\title{
Magelonidae (Polychaeta) from Hong Kong, China, with discussions on related species and redescriptions of three species
}

\author{
KATE MORTIMER ${ }^{1} \&$ ANDREW S. Y. MACKIE \\ Department of Biodiversity \& Systematic Biology, Amgueddfa Cymru - National Museum Wales, Cathays Park, Cardiff CF10 3 NP, \\ Wales,U.K.E-mail: Katie.Mortimer@museumwales.ac.uk; Andrew.Mackie@museumwales.ac.uk \\ ${ }^{1}$ Corresponding author
}

\begin{abstract}
A review of the Magelonidae from three surveys carried out in Hong Kong waters revealed the presence of three species: Magelona crenulifrons Gallardo, 1968, Magelona cornuta Wesenberg-Lund, 1949, and Magelona cf. cincta. Type materials of $M$. crenulifrons and M. cornuta were examined and redescriptions undertaken. Magelona cornuta was shown to possess a crenulate anterior margin, and the nature of the thoracic neuropodial lamellae was found to differ from the original description. Comparison with $M$. crenulifrons revealed these two species to be very morphologically similar, differing however, in several features that require close examination, including the dentition of the abdominal hooks. The presence of a species matching the description of Magelona cincta led to a redescription of the type specimen. Whilst the Hong Kong material shared many similarities with the holotype, several differences were noted. However, due to size variation between the material, the species cannot be currently described as new and is treated separately herein. Material collected from the Gulf of Oman was subsequently studied and revealed the presence of $M$. crenulifrons off Iran and Qatar, however, $M$. cornuta which was originally described from Iranian waters, was not present. Additional information has been added to species descriptions and the relationship between Indo-West Pacific species discussed.
\end{abstract}

Key words: Magelona cornuta, Magelona crenulifrons, Magelona cincta, Indo-West Pacific

\section{Introduction}

The current paper, following on from work in the Seychelles (Mortimer \& Mackie 2003, 2006), reviews the magelonids from three surveys carried out in Hong Kong, China, between 1986 and 1990. Collections were made in the northeastern Mirs Bay and Tolo Channel area in 1986 (e.g., Shin 1990), and again in 1989 (Mackie et al. 1993), while southwestern Hong Kong waters were sampled in 1990 during an ecological survey of an area to be reclaimed for airport construction. In addition, recent material from southeastern waters in the region of the Ninepin Group islands was obtained through Dr. Paul Shin (City University of Hong Kong).

Currently, nearly 70 magelonid species are recognized worldwide. Almost all are included in the genus Magelona Müller, 1858; however, two species are described for Meridithia HernándezAlcántara \& Solís-Weiss, 2000, and one for Octomagelona Aguirrezabalaga, Ceberio \& Fiege, 2001. The validity of Meridithia was recently questioned by Mortimer \& Mackie (2003).

To date, only two species of Magelona have been reported from Hong Kong waters: Magelona crenulifrons Gallardo, 1968 and an unnamed species (Mackie et al. 1993; Shin 1982 a \& b, 1998, 1990, 2003; Shin \& Thompson 1982). This review of the Hong Kong Magelonidae revealed the 
presence of Magelona cornuta Wesenberg-Lung, 1949 and a species matching the description of Magelona cincta Ehlers, 1908 in addition to the previously reported M. crenulifrons. Perceived differences from the original descriptions necessitated reexaminations of type material of all three species. The original descriptions and illustrations of these three species were found to be inadequate and in some places incorrect, therefore redescriptions of all three species were required. Differences between the type specimen of $M$. cincta and the Hong Kong material ( $M$. cf. cincta) warranted descriptions to be treated separately (see below).

\section{Materials and methods}

The majority of specimens collected in April 1986 were obtained from van Veen grab $\left(0.1-\mathrm{m}^{2}\right)$ stations surveyed by Shin (1990) in the outer Tolo Channel and Mirs Bay areas $\left(22^{\circ} 28^{\prime}-22^{\circ} 33^{\prime} \mathrm{N}\right.$, $\left.114^{\circ} 18^{\prime}-114^{\circ} 26^{\prime} \mathrm{E}\right)$, northeastern New Territories, Hong Kong, China, during the Second International Marine Biological Workshop. A station map is provided in Hirayama (1990: fig. 1). Other 1986 material was collected by, or for, Professor Christer Erséus (Göteborg University) or Dr. Akira Hirayama (Asia University, Tokyo). Maps showing the main 1986 sampling locations can be found in Hirayama (1990: fig. 1), Gibson (1990: fig. 1; region encompassing areas 7-18), and Erséus (1992: fig. 1). All samples were sieved using fine mesh sieves: 0.5-mm for grab (Shin 1990) and other macrofaunal samples (e.g., Mackie 1990) and 0.25-mm for samples taken for oligochaete specimens (Erséus 1990).

The 1990 samples were collected during a survey of southwestern waters being reclaimed for airport construction, and in associated areas likely to suffer environmental impact. Sublittoral samples were taken in the immediate vicinity of Tung Chung Wan and Chek Lap Kok, and in the borrow area north of Mo To Chau (The Brothers), north of Tai Yue Shan (Lantau Island). Grab samples were taken at 16 sites using a $0.1-\mathrm{m}^{2}$ van Veen grab and samples were subsequently sieved through coarse (1.5- or 2-mm mesh) and fine (0.5-mm mesh) sieves.

All material was initially fixed in formalin/seawater, later washed with fresh water, and preserved in $80 \%$ alcohol. With the exception of material from the Ninepin Group islands, provided by Dr. Shin, all other material was from the collections in the National Museum Wales (NMW).

Type material was borrowed from the Zoological Museum, University of Copenhagen (ZMUC) and the Museum für Naturkunde der Humboldt-Universität zu Berlin (ZMHB). Additional material of $M$. crenulifrons and $M$. cincta was borrowed from the Natural History Museum, London (BMNH).

All drawings were made using a camera lucida attachment on a Leica MZ9.5 zoom microscope, or Nikon Labophot-2 compound microscope.

To maintain consistency in the descriptions within this family, descriptions are in the same format as those used in Mortimer \& Mackie (2003, 2006). For example, specimens are herein recorded as complete (c), anterior fragments (af), posterior fragments (pf) or fragments (f), as detailed in Fiege et al. (2000). Lateral pouches on abdominal chaetigers were recorded here according to the morphologies described by Fiege et al. (2000): $\sum$ configuration, anteriorly open pouches (often convoluted) bounded dorsally and ventrally by large cuticular flaps; C configuration, posteriorly open pouches, often inconspicuous, ventral part sometimes folded over flattening pouch against body. Parapodial structures are primarily described relative to basic polychaete terminology (e.g., postchaetal, prechaetal etc.); however, certain terms introduced by Jones $(1971,1978)$ are also given (e.g., dorsal medial lobe, DML; ventral medial lobe, VML; ventral neuropodial lobe, VNL) to facilitate cross-comparisons with other publications. 


\section{Results}

Magelonidae Cunningham and Ramage, 1888

Magelona F. Müller, 1858; emended Fiege et al. (2000)

\section{Magelona cornuta Wesenberg-Lund, 1949 emended}

Figure 1

Magellone cornuta Wesenberg-Lund 1949: 328-330, fig. 36, 366, map III, 378, table I.

Magelona cornuta. Kirkegaard 1959: 26 (spelling corrected at the request of Wesenberg-Lund); Wilson 1959: 553-555, fig. 2 (redescription).

Material examined. Gulf of Oman, 20 miles E by N of Ras Jagin, Sta. $100\left(25^{\circ} 31^{\prime} 30^{\prime \prime} \mathrm{N}, 58^{\circ} 29^{\prime} \mathrm{E}\right)$, clay, 12 m, Holotype (ZMUC-POL-969; 1 af), two abdominal slide preparations (ZMUC-POL-963 pars), Petersen grab, 30 Mar 1938 (Wesenberg-Lund [1949: table I] incorrectly states position as $38^{\circ} 29^{\prime}$ E). - Hong Kong, East of Kwo Chau Kwan To (Ninepin Group islands): Stas. B4-B8 (between $22^{\circ} 14.717^{\prime} \mathrm{N}-22^{\circ} 17.978^{\prime} \mathrm{N}$ and $\left.114^{\circ} 20.165^{\prime} \mathrm{E}-114^{\circ} 25.482^{\prime} \mathrm{E}\right),(9$ af).

Diagnosis. Prostomium longer than wide, subtriangular, with distinct prostomial horns, anterior margin crenulate. Notopodia of chaetigers 1-8 with large foliaceous distally rounded postchaetal lamellae, and slender cirriform dorsal processes. Neuropodia with slender cirriform ventral processes and small postchaetal expansions that gradually increase in size before becoming large and triangular on chaetiger 8 . Chaetiger 9 notopodia with large distally pointed auricular lamellae, dorsal processes absent; neuropodia with short triangular postchaetal lobes and slender ventral prechaetal processes. Thoracic chaetigers with winged capillary chaetae. Chaetae of chaetiger 9 shorter, not otherwise modified. Abdominal lateral lamellae broadly spatulate, basally constricted. Abdominal inferior and superior processes short, triangular. Hooded hooks tridentate, in two groups, vis-á-vis. Posterior unknown.

Description. A medium-sized species; abdomen wider than thorax. Holotype incomplete, in poor condition (as previously stated in Wilson's (1959) redescription). Prostomium $1 \mathrm{~mm}$ long, 0.8 $\mathrm{mm}$ wide, thorax $5.0 \mathrm{~mm}$ long, $0.85 \mathrm{~mm}$ wide, abdomen $1 \mathrm{~mm}$ wide; total length $13 \mathrm{~mm}$ for 27 chaetigers, posterior unknown. Marked difference between thorax and abdomen, constricted at chaetiger 9. Chaetiger 7 on both sides, and chaetigers 9, 18, and 20 on left side previously dissected and absent (Fig. 1A). Wilson (1959) noted the presence of microscope preparations. On consultation with the Zoological Museum, University of Copenhagen, two slides (both labeled ZMUC-POL-963-Pars) were located and deemed to belong to the holotype. Both show abdominal parapodia and hooded hooks.

Prostomium longer than wide (L:W ratio 1.25), triangular in all material, shape slightly altered in holotype due to condition (Fig. 1B); with distinct prostomial horns, anterior margin minutely crenulated with eight irregular crenulations, eyes absent; two prominent longitudinal dorsal muscular ridges, diverging at both ends, two outer muscular ridges present either side and abutting inner pair. Proboscis everted, heart-shaped, longitudinally ridged superiorly and inferiorly. Palps arising ventrolaterally from base of prostomium, both distally incomplete, reaching around chaetiger 6; nonpapillated region long, reaching to chaetiger 3 (Fig. 1A); papillae short. Palps with three rows of papillae, either side of inconspicuous ventral groove. Palps from Hong Kong specimens long, reaching chaetiger 14+, nonpapillated region reaching chaetiger 3-4; papillae initially short, increasing in length around chaetiger 5 . 


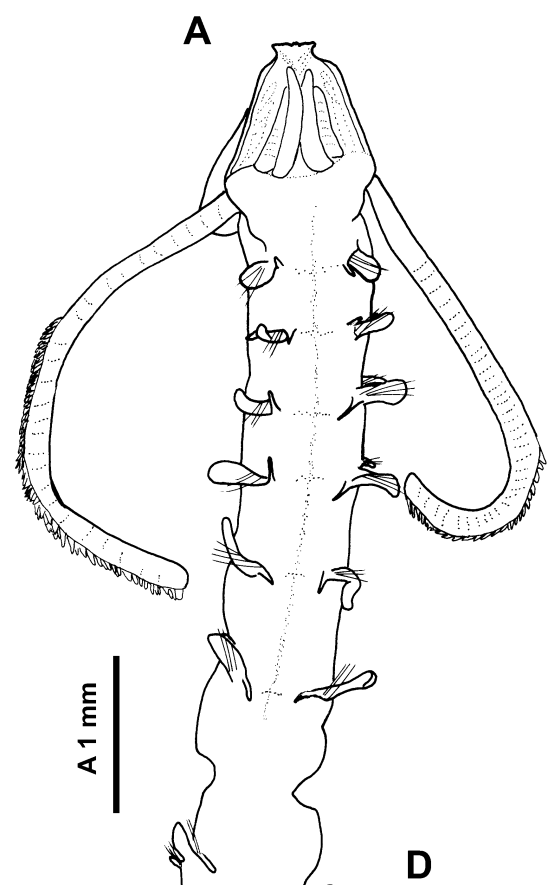

D
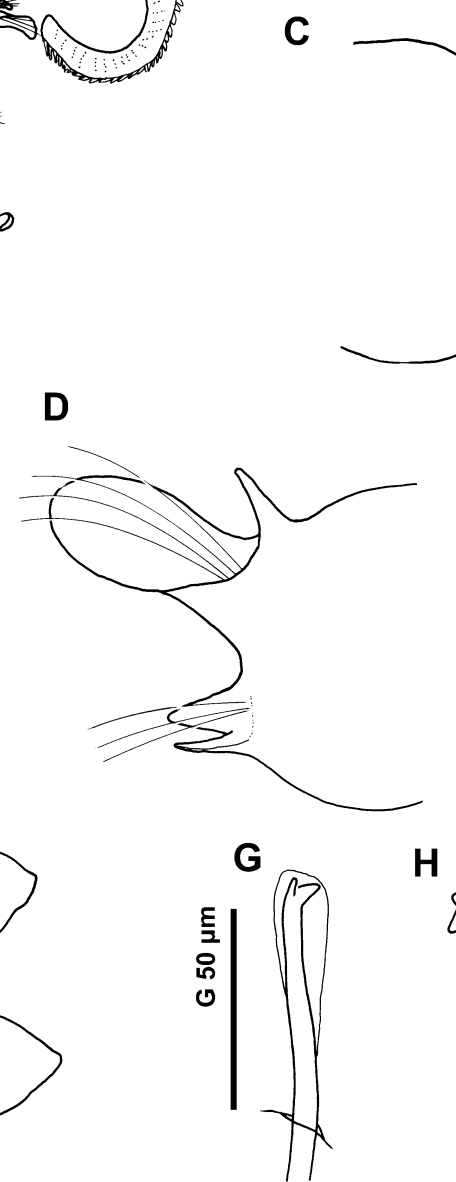

$\mathbf{F}$

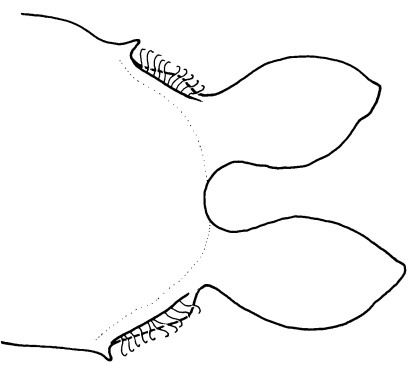

H
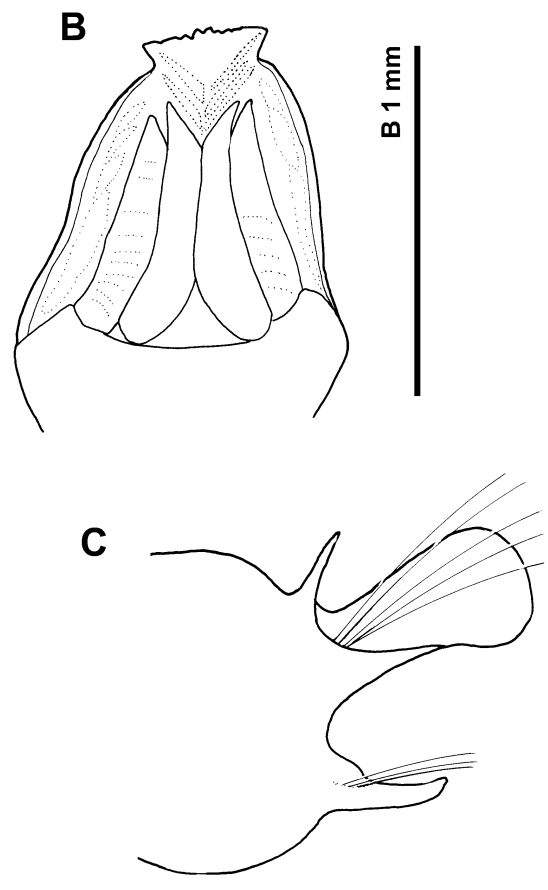

E
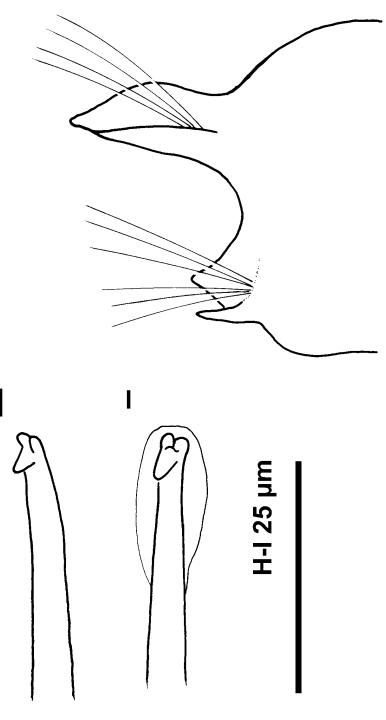

I
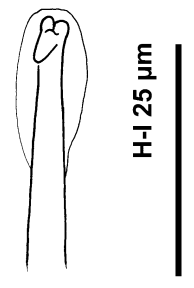

FIGURE 1. Magelona cornuta (Holotype, ZMUC-POL-969). A, anterior region, dorsal view; B, prostomium, dorsal view; C-F, chaetigers 5, 8, 9, and 17 respectively, anterior views; G-I, tridentate hooded hooks, lateral and oblique frontal views. 
Peristomium achaetous, of roughly equal size to chaetiger 1 . Chaetigers $1-8$ similar (Figs. 1C, D); parapodia biramous with triangular notopodial prechaetal lamellae confluent with large foliaceous and distally rounded postchaetal lamellae, latter shortest on chaetiger 1 and of similar length on chaetigers 2-6 (lobes of chaetiger 7 missing on holotype, previously dissected). Single long, slender, tapering cirriform prechaetal superior processes (DML) present, increasing in length from chaetigers 1-3, and of similar length on chaetigers 4-8. Neuropodia with single slender cirriform processes ventrally (VNL), directly under chaetae and decreasing in size throughout thorax, confluent with low prechaetal ridges. Single short and rounded postchaetal lobe just visible on chaetigers 2 and 3, apparently becoming broader and lower, continuous with ventral processes on chaetigers 4-6; exact nature difficult to discern due to condition of holotype. Chaetiger 7 previously dissected, neuropodia unknown. Chaetiger 8 (Fig. 1D) with neuropodial ventral processes directly under chaetae, but slightly prechaetal in position, similar in length to conspicuous triangular postchaetal lamellae.

Chaetiger 9 (Fig. 1E) with notopodia having large ear-like postchaetal lamellae, shorter than on preceding chaetigers, distal tip pointing ventrally; prechaetal lamellae low, triangular, no superior processes. Neuropodial postchaetal lamellae more triangular, with slender ventral processes in distinctly prechaetal position. All thoracic chaetae simple winged capillaries, shorter on chaetiger 9.

Abdominal chaetigers with broad spatulate lateral lamellae, of about equal size in both rami, basally constricted, initially rounded and subsequently somewhat bluntly pointed (Fig. 1F). Lateral lamellae extend postchaetally as rounded ridges. Small triangular processes (DML \& VML) present at inner margins of chaetal rows.

Abdominal chaetae tridentate hooded hooks of similar size, superior two fangs parallel, in close proximity to each other, above main fang (Figs. 1G-I), an occasional bidentate hook observed in some Hong Kong specimens. When present, secondary tooth of bidentate forms much larger than those of tridentate hooks. Hooks in two equal groups, main fangs vis-á-vis. Approximately 8-10 hooks per ramus. Small curved support chaetae present, one per ramus, basal regions overlapping in area between notopodial and neuropodial rami of each parapodium; distal regions small with rudimentary hook emerging at bases of lateral lamellae.

No pouches on anterior abdominal segments of holotype. Posterior chaetigers unknown. Hong Kong specimens incomplete but with paired, consecutively occurring C-configuration posteriorly open pouches starting around chaetiger 41 . Pouches differing from previously observed simple Cconfiguration pouches, with a medial slit separating dorsal and ventral halves. Slit surrounded by a thick cuticle.

Color. Preserved specimens cream in alcohol. Intense glandular areas on abdominal chaetigers as interparapodial patches. Some Hong Kong specimens stained with Rose Bengal reveal dorsal thoracic traverse stripes, especially between chaetigers 3-8, often with a large pink spot around chaetiger 1; abdominal segments with interparapodial patches, especially in anterior abdomen. Staining with methyl green diffuse, no clear pattern on holotype or Hong Kong specimens.

Habitat. Type specimen found in clay, $12 \mathrm{~m}$, Gulf of Oman. Other material from five sublittoral stations in southeastern Hong Kong waters.

Remarks. Wilson (1959) re-examined the holotype, emending Wesenberg-Lund's original description, with particular reference to the neuropodia of chaetiger 8 and the tridentate nature of the abdominal hooks. In both the original description and Wilson's re-examination, there was no mention of a crenulate prostomial anterior margin as observed here. Wilson, describing chaetiger 8 , stated that "the neuropodial bristles spring between two finger-like processes the lower one being the ventral cirrus." These are what the present authors describe as a triangular postchaetal lamella (not mentioned in the original description) and a neuropodial ventral process. Neither description 
mentions any slight postchaetal expansions on the neuropodia of chaetigers 2-6 or the ventral prechaetal process on chaetiger 9 . The exact shape and form of chaetiger 7 cannot be detailed; however, the notopodial lamellae are likely to be of similar shape and size to those on chaetigers 6-8 due to the uniformity of thoracic chaetigers (and from observations on the Hong Kong material). As Wilson stated, tridentate abdominal hooded hooks are present; Wesenberg-Lund's original description of "blunt bidentate tip" and her drawings may be a posterior view of a tridentate hook, where the main fang is obscured.

Magelona cornuta most closely resembles M. crenulifrons Gallardo, 1968 from Vietnam and Magelona sp. G of Uebelacker \& Jones (1984) from the Gulf of Mexico. Both have longer-thanwide prostomia with well-developed frontal horns and can have crenulations on a relatively straight anterior prostomial margin.

Magelona sp. G approaches M. cornuta in many parapodial and chaetal features. Magelona sp. G differs most markedly in having bidentate abdominal hooks and in possessing low postchaetal notopodial lamellae on chaetiger 9. Paired posteriorly open lateral pouches are present from chaetiger 26-27 onward.

Seven additional species with frontal horns and anterior prostomial crenulations differ from $M$. cornuta in having prostomia with widths equal to, or greater than, their lengths. In addition, the anterior margins of their prostomia tend to be rounded or triangular, rather than straight. Of these, $M$. tehuanensis Hernández-Alcántara \& Solís-Weiss, 2000 from western Mexico most resembles $M$. cornuta, but has a rounded anterior prostomium and possesses low notopodial postchaetal lamellae on chaetiger 9.

Magelona sp. L of Uebelacker \& Jones (1984) from the Gulf of Mexico differs in having a rounded anterior prostomium, broad triangular dorsal thoracic processes, and more angular thoracic notopodial lamellae, while lacking (?) a neuropodial postchaetal lobe on chaetiger 9. Paired posteriorly open lateral pouches are present from chaetiger 28-31 onward.

Magelona lenticulata Gallardo, 1968 from Vietnam differs in having a broadly rounded to triangular anterior prostomial margin, short broad dorsal processes in the anterior thorax and small digitiform dorsal processes on chaetiger 9. Magelona petersenae Nateewathana \& Hylleberg, 1991 and M. methae Nateewathana \& Hylleberg, 1991 resemble M. lenticulata in prostomial shape. The first further differs from $M$. cornuta in having slender triangular notopodial postchaetal lamellae and in lacking dorsal processes. The second is readily distinguished by having broad dorsal notopodial processes and bidentate abdominal hooks.

Magelona longicornis Johnson, 1901 from NW North America (as redescribed by Jones 1971) does have a more truncate prostomium, but differs markedly from all the above in having bidentate unidirectional abdominal hooks and in lacking dorsal processes on chaetiger 9. Furthermore, the postchaetal lamellae of the thoracic notopodia and abdominal parapodia are more slender and tapered.

A seventh species, Magelona sp. J of Uebelacker \& Jones (1984), has also been reported to sometimes have crenulations on the anterior prostomium. However, the prostomium is again broad and the frontal horns were described as rudimentary, the anterior thoracic dorsal processes are broadly conical, and dorsal processes are present on chaetiger 9.

Magelona cornuta had been previously recorded from the Red Sea (Amoureux 1983), Gulf of Aden (Hartman 1974, 1976), Portugal (J. Gil), and the Ivory Coast (Intes \& Le Loeuff 1975). Kirkegaard (1959) recorded it from the west coast of Africa (off Nigeria to off Angola), mostly shallow 19-75 m, but one station off N Angola was at $308 \mathrm{~m}$. Kirkegaard checked the type and reported his specimens to be "quite identical," although due to the inaccuracies in the original description it is not known whether these records are correct. The specimens collected off Portugal 
by J. Gil have been examined and deemed to be a morphologically similar but differing species (pers. obs). Amoureux (1983) said his specimen, an anterior fragment with very marked frontal horns, without modified chaetae on the 9th chaetiger agreed in all respects with the details and corrections given by Wilson (1959) on his reexamination of the type specimen. Until material from other areas can be examined, we consider M. cornuta to have an Indo-West Pacific distribution.

\section{Magelona crenulifrons Gallardo, 1968}

Figures 2-4

Magelona crenulifrons Gallardo 1968: 100 \& 238-239, pl. XLIV, figs. 1-5.

Material examined. Vietnam, Bay of Nha Trang, Sta. $96\left(\sim 12^{\circ} 11^{\prime} 20^{\prime \prime} \mathrm{N}, 109^{\circ} 13^{\prime} 40^{\prime \prime} \mathrm{E}\right.$, near Hòn Miêũ island), mud, 9 m, paratype (ZMUC-POL-1416; af), van Veen, 5 Feb 1960; Sta. 100 I $\left(\sim 12^{\circ} 11^{\prime} 05^{\prime \prime} \mathrm{N}, 109^{\circ} 13^{\prime} 30^{\prime \prime} \mathrm{E}\right.$, near Hòn Miêũ island), mud, 14 m, paratype (ZMUC-POL-1417; 2 f), van Veen, 5 Feb 1960; same locality, paratype (ZMUC-POL-1418; af); Sta. 101 II ( 12 $11^{\prime} 00^{\prime \prime} \mathrm{N}$, $109^{\circ} 13^{\prime} 50^{\prime \prime} \mathrm{E}$, between Hòn Miêũ and Hòn Tâ'm islands), mud, 14 m, paratype (ZMUC-POL-1419; 2 af), van Veen, 5 Feb 1960; Sta. 159 II ( 12 $\left.19^{\prime} 20^{\prime \prime} \mathrm{N}, 109^{\circ} 15^{\prime} 40^{\prime \prime} \mathrm{E}\right)$, mud, $16 \mathrm{~m}$, paratype (ZMUC-POL-1420; af, 2 pf), Petersen grab, 24 Feb 1960; Sta. 283 ( 12 10'30"N, 109 16'50"E, south Hòn L'õn), mud, 21 m, paratype (ZMUC-POL-1421; af), van Veen, 23 Mar 1960. Note latitudes and longitudes estimated from Gallardo (1968: 38, chart 1).- - Hong Kong (NE), Chek Mun Hoi Hap (Tolo Channel): Sta. 47 (Hirayama 1990: fig. 1), sandy mud, 21 m (NMW.Z. 1986.079.0170; af, f, 1 pf), coll. A.S.Y. Mackie 14 Apr 1986; Sta. 48, sandy mud, 20 m (NMW.Z. 1986.079.172; af), coll. A. Hirayama 14 Apr 1986; Hoi Ha Wan (Jones Cove), sandy, 6 m (NMW.Z.1986.079.0168; 2 af, 4 f), coll. A. Hirayama 6 Apr 1986.-Tai Pang Wan (Mirs Bay), east of Ngo Mei Chau (Crescent Island), Sta. 14, fine silt/clay, >10 m (NMW.Z.1986.079.0174; af), coll. A. Hirayama 6 Apr 1986; E by N of Chek Chau (Port Island), Sta. 21, fine silt/clay, 17 m (NMW.Z. 1986.079.0169; af), coll. A.S.Y Mackie 8 Apr 1986; same locality (NMW.Z.1986.079. 0173; 2 af), coll. A. Hirayama 8 Apr 1986; ENE of Chek Chau (Port Island), Sta. 22, fine silt/clay, $17 \mathrm{~m}$ (NMW.Z. 1986.079.0175; af), coll. A.S.Y Mackie 8 Apr 1986; NE of Chek Chau (Port Island), Sta. 23, fine silt/clay, 18 m (NMW.Z. 1986.079.0171; af), coll. A.S.Y. Mackie 8 Apr 1986.—Hong Kong (SW), west of Chek Lap Kok (now HK International Airport), Sta. 10B (22 $\left.{ }^{\circ} 18.1^{\prime} \mathrm{N}, 113^{\circ} 55.2^{\prime} \mathrm{E}\right)$, mud and shell gravel, 2 m (NMW.Z.2007.033.0001; af), coll. P. Rainbow \& J. Taylor 6 Nov 1990. Sta. 8B (22 $\left.2^{\circ} 17.9^{\prime} \mathrm{N}, 113^{\circ} 53.0^{\prime} \mathrm{E}\right)$, mud, 6 m (NMW.Z.2007.033.0002; af), coll. P. Rainbow \& J. Taylor 6 Nov 1990.- Iran (offshore; BMNH material), Sta. $1\left(26^{\circ} 59^{\prime} \mathrm{N}, 52^{\circ} 29^{\prime} \mathrm{E}\right)$, poorly sorted fine silt, $70 \mathrm{~m}$ (6 af, $9 \mathrm{f})$; Sta. $2\left(26^{\circ} 58^{\prime} \mathrm{N}, 52^{\circ} 30^{\prime} \mathrm{E}\right)$, poorly sorted fine silt, $70 \mathrm{~m}$ (3 af, $\left.1 \mathrm{f}\right)$; Sta. 3 $\left(26^{\circ} 59^{\prime} \mathrm{N}, 52^{\circ} 30^{\prime} \mathrm{E}\right)$, poorly sorted fine silt, $70 \mathrm{~m}(2 \mathrm{af}, 1 \mathrm{f})$; Sta. $4\left(26^{\circ} 60^{\prime} \mathrm{N}, 52^{\circ} 29^{\prime} \mathrm{E}\right)$, poorly sorted fine silt, $69.8 \mathrm{~m}$ (4 af, $1 \mathrm{f})$; Sta. $5\left(27^{\circ} 00^{\prime} \mathrm{N}, 52^{\circ} 29^{\prime} \mathrm{E}\right)$, poorly sorted fine silt, $69.4 \mathrm{~m}$ (2 af); Sta. 6 $\left(27^{\circ} 00^{\prime} \mathrm{N}, 52^{\circ} 29^{\prime} \mathrm{E}\right)$, very poorly sorted coarse silt, $69.5 \mathrm{~m}(2 \mathrm{af}, \mathrm{f})$; Sta. $8\left(27^{\circ} 00^{\prime} \mathrm{N}, 52^{\circ} 28^{\prime} \mathrm{E}\right)$, very poorly sorted fine silt, $68.5 \mathrm{~m}$ (4 af, $5 \mathrm{f})$; Sta. $9\left(26^{\circ} 59^{\prime} \mathrm{N}, 52^{\circ} 28^{\prime} \mathrm{E}\right)$, very poorly sorted medium silt, $69.4 \mathrm{~m}$ (4 af, $1 \mathrm{f})$; Sta. $10\left(26^{\circ} 59^{\prime} \mathrm{N}, 52^{\circ} 29^{\prime} \mathrm{E}\right)$, poorly sorted fine silt, $69.5 \mathrm{~m}(2 \mathrm{af})$; Sta. $14\left(26^{\circ} 60^{\prime} \mathrm{N}\right.$, $\left.52^{\circ} 30^{\prime} \mathrm{E}\right)$, poorly sorted fine silt, $69.6 \mathrm{~m}(2 \mathrm{af}, 2 \mathrm{f})$; Sta. $15\left(26^{\circ} 56^{\prime} \mathrm{N}, 52^{\circ} 26^{\prime} \mathrm{E}\right)$, very poorly sorted fine silt, $70.0 \mathrm{~m}(6 \mathrm{af}, 2 \mathrm{f})$ : Sta. $17\left(26^{\circ} 53^{\prime} \mathrm{N}, 52^{\circ} 29^{\prime} \mathrm{E}\right)$, very poorly sorted very fine sand, $69.9 \mathrm{~m}(3$ af); Sta. $18\left(26^{\circ} 53^{\prime} \mathrm{N}, 52^{\circ} 28^{\prime} \mathrm{E}\right)$, very poorly sorted medium silt, $74.5 \mathrm{~m}$ (5 af, $\left.1 \mathrm{f}\right)$; Sta. $19\left(26^{\circ} 53^{\prime} \mathrm{N}\right.$, $\left.52^{\circ} 28^{\prime} \mathrm{E}\right)$, very poorly sorted fine silt, $73.5 \mathrm{~m}(4 \mathrm{af})$; Sta. $20\left(26^{\circ} 54^{\prime} \mathrm{N}, 52^{\circ} 28^{\prime} \mathrm{E}\right)$, poorly sorted fine silt, $72.5 \mathrm{~m}$ ( $8 \mathrm{af}, 2 \mathrm{f})$; Sta. $21\left(26^{\circ} 54^{\prime} \mathrm{N}, 52^{\circ} 28^{\prime} \mathrm{E}\right)$, poorly sorted fine silt, $71.6 \mathrm{~m}$ (3 af $)$; Sta. 23 $\left(26^{\circ} 55^{\prime} \mathrm{N}, 52^{\circ} 27^{\prime} \mathrm{E}\right)$, very poorly sorted coarse silt, $69.0 \mathrm{~m}(7 \mathrm{af})$; Sta. $24\left(26^{\circ} 53^{\prime} \mathrm{N}, 52^{\circ} 27^{\prime} \mathrm{E}\right)$, very 
poorly sorted very fine sand, $68.5 \mathrm{~m}(1 \mathrm{af})$; Sta. $25\left(26^{\circ} 53^{\prime} \mathrm{N}, 52^{\circ} 28^{\prime} \mathrm{E}\right)$, very poorly sorted medium silt, $73.9 \mathrm{~m}$ (11 af, $8 \mathrm{f})$; Sta. $26\left(26^{\circ} 53^{\prime} \mathrm{N}, 52^{\circ} 28^{\prime} \mathrm{E}\right)$, poorly sorted fine silt, $73.3 \mathrm{~m}$ (4 af, $\left.4 \mathrm{f}\right)$; Sta. 27 $\left(26^{\circ} 54^{\prime} \mathrm{N}, 52^{\circ} 28^{\prime} \mathrm{E}\right)$, very poorly sorted fine silt, $71.7 \mathrm{~m}(4 \mathrm{af}, 3 \mathrm{f})$; Sta. $28\left(26^{\circ} 54^{\prime} \mathrm{N}, 52^{\circ} 28^{\prime} \mathrm{E}\right)$, poorly sorted fine silt, $71.7 \mathrm{~m}$ (5 af, $3 \mathrm{f})$; Sta. $30\left(26^{\circ} 54^{\prime} \mathrm{N}, 52^{\circ} 38^{\prime} \mathrm{E}\right)$, poorly sorted fine silt, $72.2 \mathrm{~m}$ (2 af, 3 f); - Iran (nearshore; BMNH material), Sta. $31\left(27^{\circ} 42^{\prime} \mathrm{N}, 52^{\circ} 18^{\prime} \mathrm{E}\right)$, coarse silt, $16.0 \mathrm{~m}$ (3 af); Sta. 35 $\left(27^{\circ} 41^{\prime} \mathrm{N}, 52^{\circ} 18^{\prime} \mathrm{E}\right)$, medium silt, $21.0 \mathrm{~m}(2 \mathrm{af}) ;$ Sta. $47\left(27^{\circ} 38^{\prime} \mathrm{N}, 52^{\circ} 18^{\prime} \mathrm{E}\right)$, very fine silt, $48.0 \mathrm{~m}(3$ af).-Q Qatar off northern coast $\left(26^{\circ} 38^{\prime} \mathrm{N}, \sim 51^{\circ} 40^{\prime} \mathrm{E}\right.$; BMNH material), Sta. 2 (1 af); Sta. 5 (1 af); Sta. 6 (4 af, 2 f); Sta. 9 (2 af); Sta. 11(1 af).

Diagnosis. Prostomium slightly longer than wide, spatulate, with distinct frontal horns, anterior margin crenulate. Notopodia of chaetigers 1-8 with large foliaceous postchaetal lamellae, and slender cirriform dorsal processes. Neuropodia with slender cirriform ventral processes. Chaetiger 9 with large distally pointed auricular lamellae, dorsal processes absent; neuropodia with short triangular postchaetal lobes and slender ventral prechaetal processes. Thoracic chaetigers with winged capillary chaetae. Abdominal lateral lamellae broadly spatulate, basally constricted. Abdominal inferior and superior processes short, triangular. Hooded hooks bidentate, in two groups, vis-á-vis. C-configuration lateral pouches present abdominally.

Description. A moderately sized species, lightly constricted at chaetiger 9, all paratypes incomplete. Largest observed paratype (ZMUC-POL-1419) prostomium $0.6 \mathrm{~mm}$ long, $0.4 \mathrm{~mm}$ wide, thorax (including prostomium) $3.1 \mathrm{~mm}$ long, $0.5 \mathrm{~mm}$ wide, posterior $0.5 \mathrm{~mm}$ wide; body length $25 \mathrm{~mm}$ for 50 chaetigers. Holotype not available for observation. Observed paratype material generally in poor condition, epidermis separating from lower layers in all specimens (e.g., Fig. 4A: in this specimen separation noticeable between chaetigers 1 and 4, marked by curved dotted lines between parapodia).

Prostomium slightly longer than wide (L:W ratio 1.2-1.5), rounded triangular with distinct frontal horns, anterior margin crenulate, with 6-9 crenulations (Figs. 2A-B). Shape of prostomium of type material (Figs. 4A-B) appearing more triangular due to poor condition of specimen, lateral edges compressed. Two prominent dorsal longitudinal muscular ridges, extending into frontal horns, diverging anteriorly, two outer muscular ridges either side, abutting inner pair. Distinct muscular pattern either side of ridges as curved lines. Proboscis heart-shaped, with distinct longitudinal ridges. Palps absent on all type and Hong Kong material, however, palps present on Iran material; long, slender reaching chaetiger 25+, nonpapillated region reaching approximately chaetiger 3 .

Peristomium achaetous, slightly longer than chaetiger 1 . Notopodia of chaetigers 1-8 similar, with large foliaceous and distally rounded postchaetal lamellae gradually increasing in size along thorax, inferiorly encircling chaetae, forming low triangular prechaetal lamellae (Figs. 2C-E, 4C-E). Superiorly, single long, slender processes (DML) present. Neuropodia of chaetigers 1-8 similar, with slender cirriform ventral neuropodial lobes (VNL) decreasing gradually in size along thorax, and slight postchaetal expansion. Chaetiger 8 (Figs. 2E, 4E) with conspicuous triangular postchaetal lamellae and single small slender cirriform ventral neuropodial lobes (sometimes difficult to see due to size).

Chaetiger 9 (Figs. 2F, 4F) notopodial postchaetal lamellae shorter than those of preceding chaetigers, auricular, with small distal tips, prechaetal lamellae low triangular, superior prechaetal processes absent. Neuropodial postchaetal lamellae triangular, with slender ventral processes. All thoracic chaetae simple winged capillaries.

Abdomen with basally constricted lateral lamellae, large rounded spatulate, distally pointed, in both rami (Figs. 2G-H, 4G). Hooks protruding from definite ridge. Short triangular processes (DML \& VML) evident throughout at inner margins of chaetal rows. C-configuration pouches, posteriorly open, unpaired, on alternate chaetigers more or less regularly, observed in both the type and Hong 

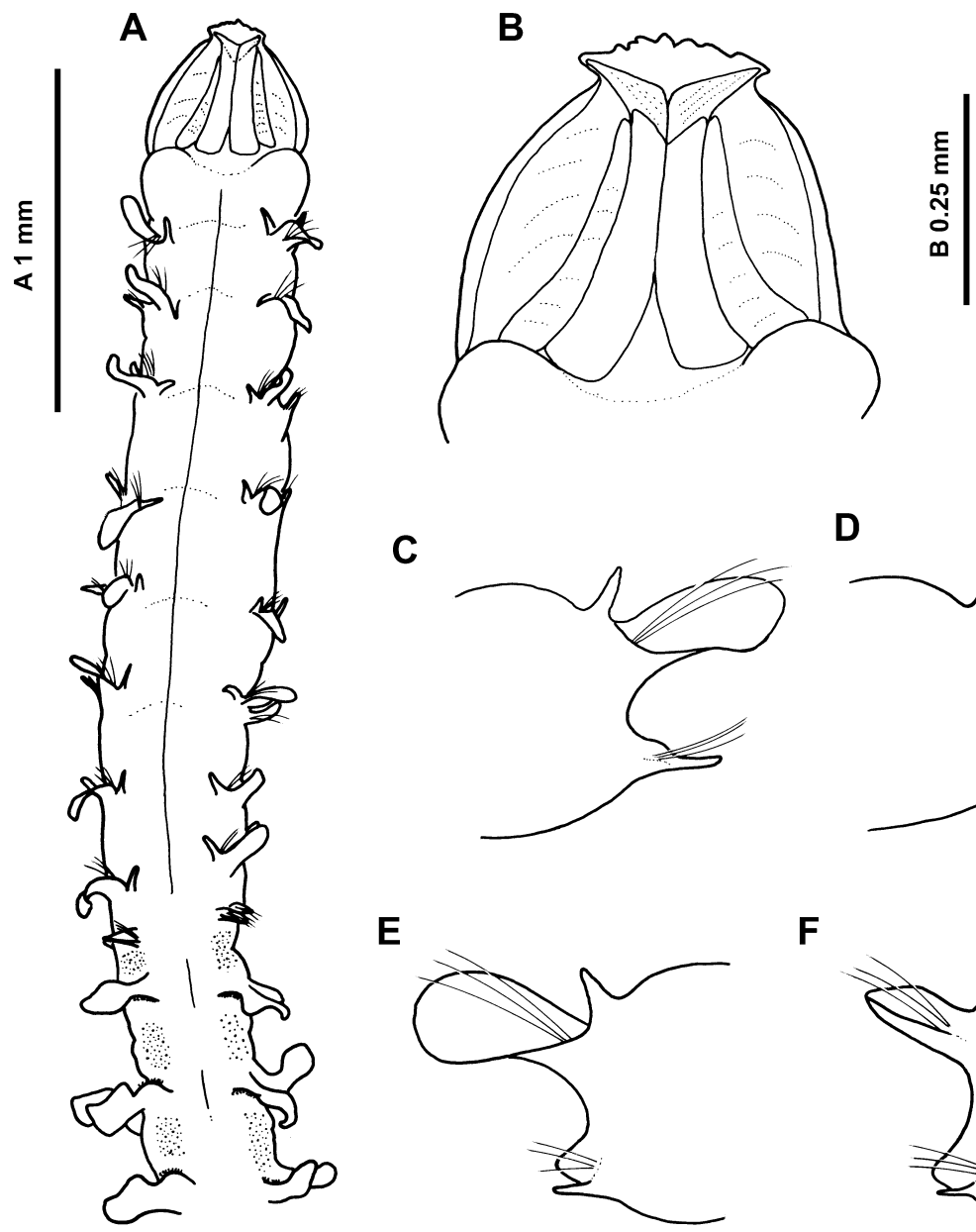

C

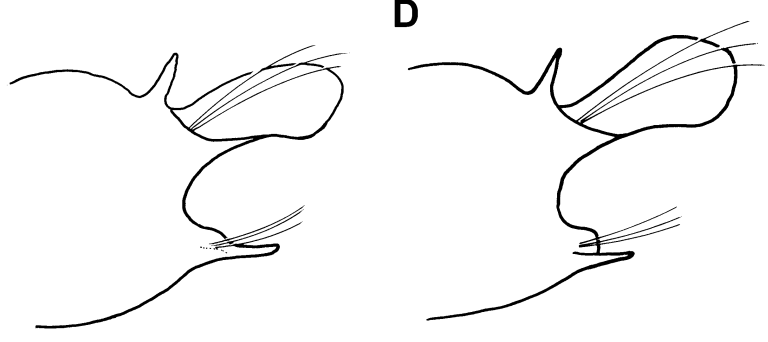

E

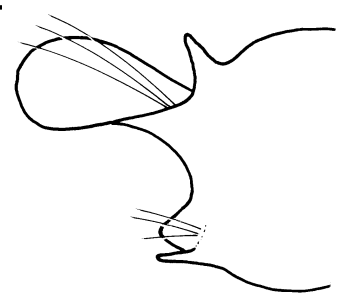

$\mathbf{F}$

H
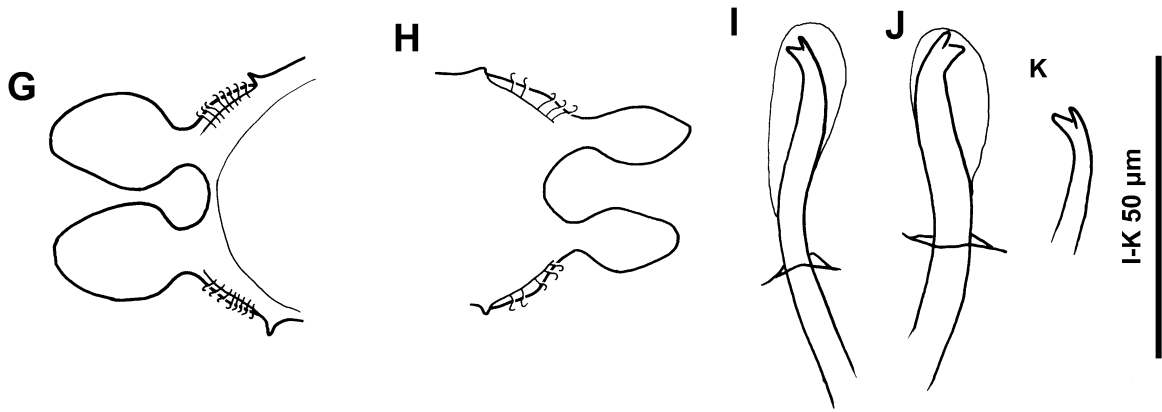

FIGURE 2. Hong Kong Magelona crenulifrons (anterior and prostomium NMW.Z.2007.033.0001, parapodia NMW.Z.1986.079.0170). A, anterior region, dorsal view; B, prostomium, dorsal view; $\mathrm{C}-\mathrm{H}$, chaetigers 5, 7, 8, 9 , 10 and 22 respectively, anterior views; I-K, bidentate hooded hooks, lateral and oblique rear views. 


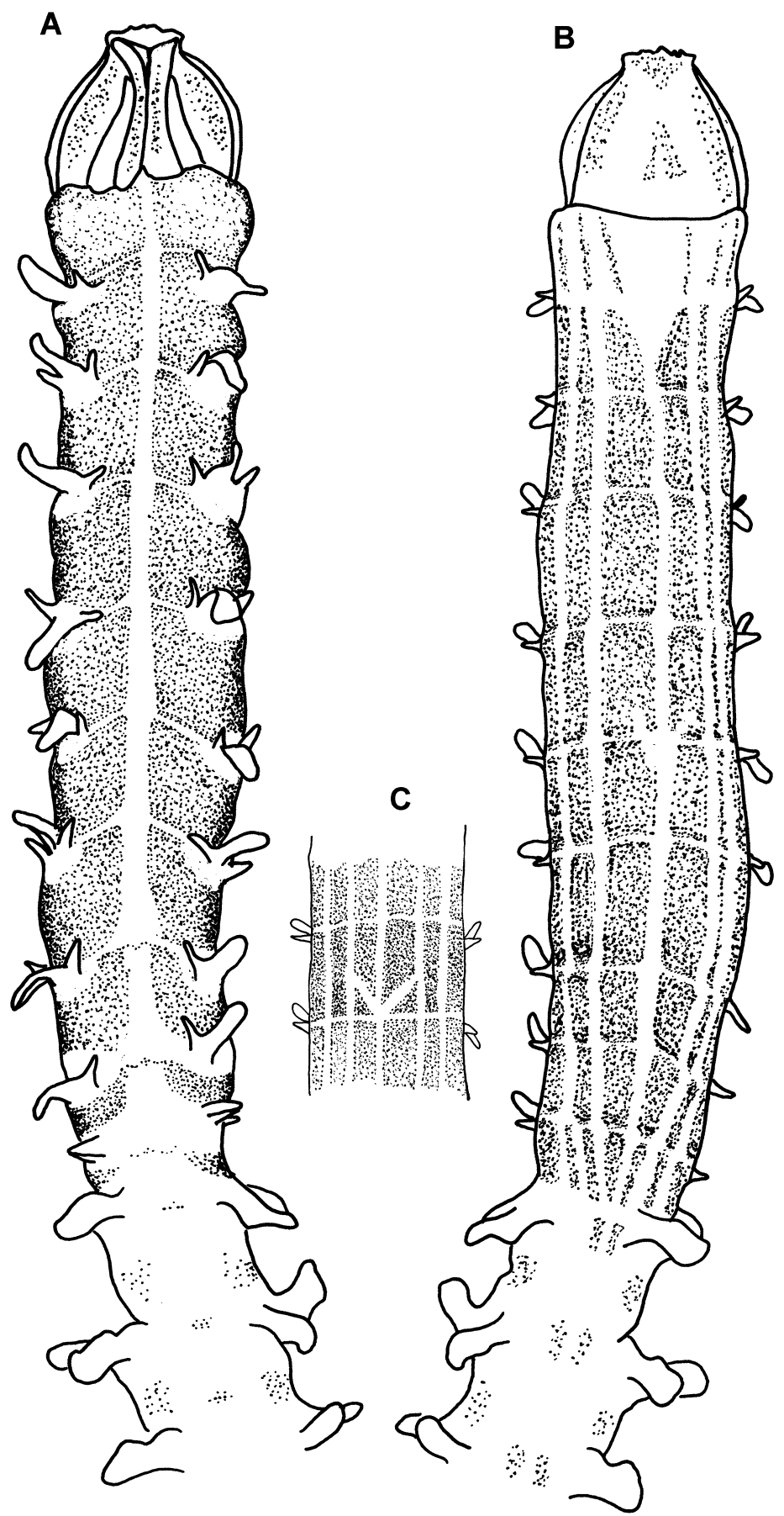

FIGURE 3. Hong Kong Magelona crenulifrons anterior, methyl green staining pattern. A, dorsal view; B, ventral view; C, variation in ventral V-shaped pattern around chaetiger 5. 

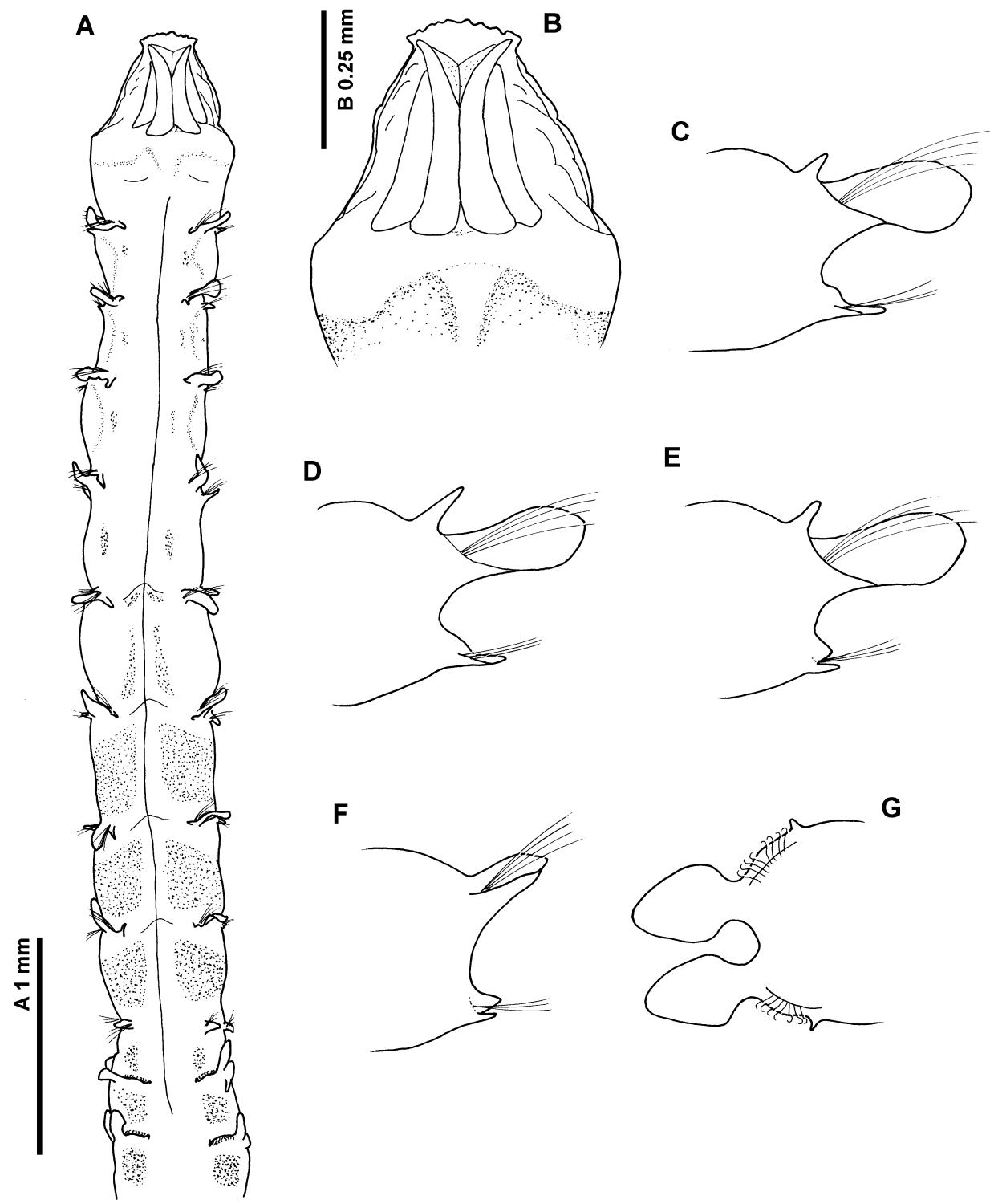

D

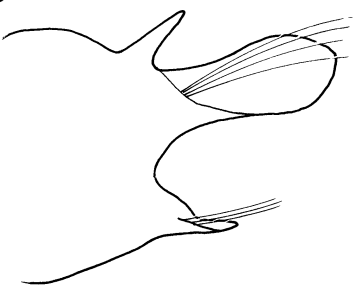

E

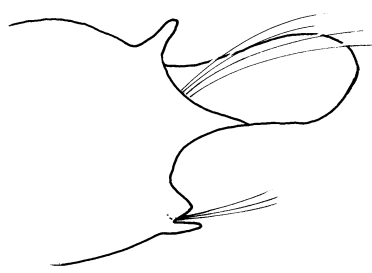

$\mathbf{F}$
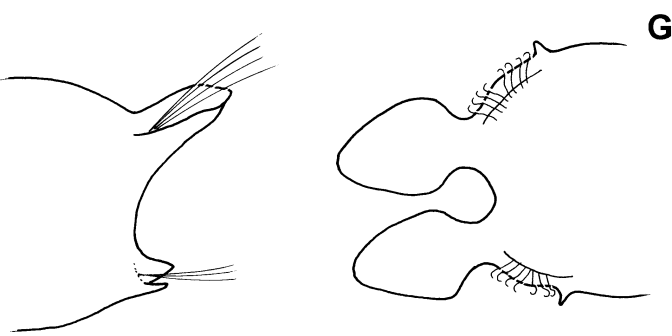

FIGURE 4. Magelona crenulifrons (Paratype, ZMUC-POL-1421; paratype, ZMUC-POL-1416 chaetigers 8 \& 11). A, anterior region, dorsal view, showing methyl green staining pattern; B, prostomium, dorsal view; $\mathrm{C}-\mathrm{G}$, chaetigers $2,4,8,9$, and 11 respectively, anterior views.

Kong material (although not reported by Gallardo). Hong Kong specimen from Station 23 (NMW.Z. 1986.079.0171) with pouches on the following segments; 25R, 27R, 28L, 29R, 30L, 31L, 31R, 32L, 
33L, 33R, 35L, 35R, 36L, 37R, 38L, 38R, 39R, 40L, 42L, 42R, 43L, 43R, 44L, 44R, 45L \& 45R. Pygidium unknown.

Abdominal hooded hooks strongly bidentate, secondary tooth separated from main fang by large cleft (Figs. 2I-K); secondary tooth larger than the two secondary teeth observed in M. cornuta. Hooks in two groups, vis-á-vis, initially 8-10 per ramus. Small curved support chaetae present, one per ramus, basal areas overlapping in region between notopodial and neuropodial rami of each parapodium; distal regions small with rudimentary hook, emerging at bases of lateral lamellae, similar to those seen in Magelona cornuta.

Color. No live specimens observed. All specimens preserved in alcohol, cream-white in color. Iran and Qatar specimens stained with Rose Bengal; dorsal thoracic traverse stripes between parapodia, continuing into abdominal segments. Ventral stripes only apparent in posterior thorax, but continue into abdomen, ventral stripes less obvious than dorsal ones. Very intense interparapodial patches, especially in the anterior abdomen.

Methyl green staining pattern conspicuous (Figs. 3, 4A). Dorsal surface speckled all over, apart from mid-dorsal line and surrounding parapodia, slightly less in posterior thorax. Venter striped longitudinally, stripes narrowing to a point at the thoracic-abdominal junction. Staining slightly more diffuse dorsally on paratype material, but this decreased uptake of methyl green may be caused by condition of specimens. Ventrally, staining pattern clear in type material. Characteristic unstained medial V-shape observed in majority of specimens, including type material, point of the $\mathrm{V}$ often at junction between chaetigers 5 and 6, though some variation occurred (Figs. 3B, C).

Habitat. Paratypes of $M$. crenulifrons were examined from 20 Vietnamese stations. Other type specimens (from Stas. 44, 67, 80, 106II, 109II, 122II, 124, 130, 131, 134II, 139I, 141II, 263, 274, 355 not examined). Type material found in mud and sandy mud $(6-48 \mathrm{~m})$.

The species is additionally reported herein from ten Hong Kong stations with similar mud and muddy sand sediments (2-21 m); 34 stations off the coast of Iran (five nearshore, 29 offshore) in fine silt to coarse sand, 9-74.5 m (not all material seen); and five stations off the north coast of Qatar.

Remarks. Magelona crenulifrons has many similarities with M. cornuta, but differs notably in the nature of the hooded hooks. The other main differences separating the two species are a narrower, more triangular prostomium in $M$. cornuta (broader, more laterally rounded in $M$. crenulifrons), the presence of a ventral V-shaped nonstaining pattern in $M$. crenulifrons (not observed in $M$. cornuta), and the more anterior occurrence of the C-configuration pouches in M. crenulifrons. Magelona crenulifrons has been previously recorded from the Natuna Islands, South China Sea (AlHakin \& Glasby 2004), Thailand (Nateewathana \& Hylleberg 1991; Hylleberg \& Nateewathana 1991), and Hong Kong (Shin 1998, 2003).

\section{Magelona cincta Ehlers, 1908}

Figure 5

Magelona cincta Ehlers 1908: 111-112, pl. XV, figs. 9-12; Wilson 1958: 620-625, figs. 2 \& 3.

Non Magelona cincta Kirkegaard (1959)

Material examined. South Africa, Algoa Bay, Sta. 101 ( $\left.33^{\circ} 50^{\prime} \mathrm{S}, 25^{\circ} 48^{\prime} \mathrm{E}\right)$ among mollusks, bryozoans, and echinoderms, 40 m, Holotype (ZMHB 4531; 1 af).-Mozambique (Portuguese East Africa), Morrumbena Estuary, Linga, weed beds (BMNH.1955.4.1.96. University Cape Town ecological survey MOR.125.Q; af, f), shore, coll. University of Cape Town, 15 July 1954 (see Remarks, below). 


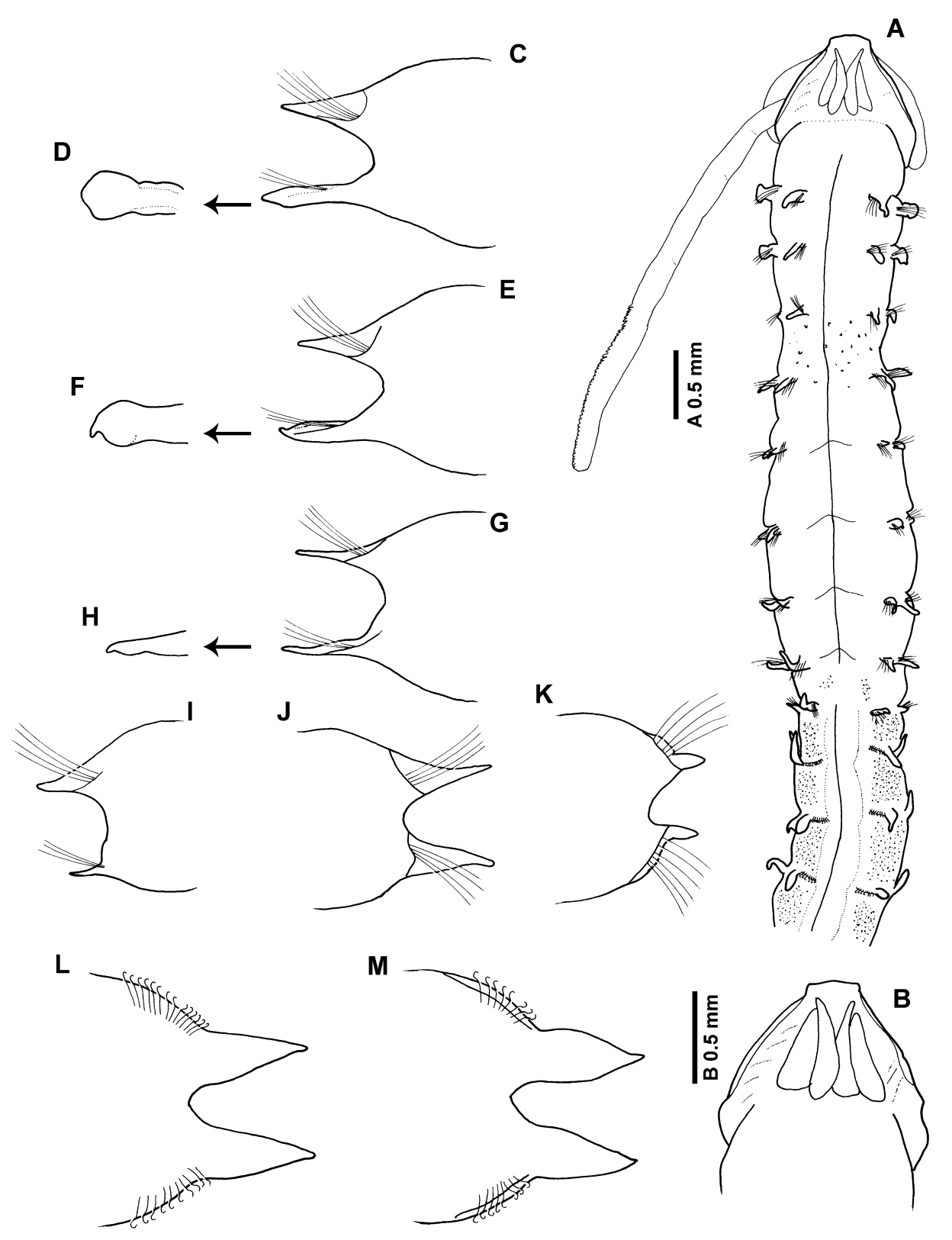

FIGURE 5. Magelona cincta (Holotype, ZMHB 4531). A, Anterior region, dorsal view; B, prostomium, dorsal view; $\mathrm{C}$, chaetiger 1 , anterior view; $\mathrm{D}$, neuropodial lamellae chaetiger 1, dorsal view; $\mathrm{E}$, chaetiger 2, anterior view; F, neuropodial lamellae chaetiger 2, dorsal view; G, chaetiger 4, anterior view; H, neuropodial lamellae chaetiger 4, dorsal view; I-M, chaetigers $6,8,9,10$, and 13 , anterior views. 
Diagnosis. A stout species. Prostomium as wide as long, sub-triangular, with rudimentary horns. Chaetigers 1-8 with slender, smooth-edged, triangular notopodial postchaetal lamellae with pointed tips, no superior processes present. Neuropodia with ventral neuropodial lamellae, expanded distally between chaetigers 1-4, lamellae postchaetal by chaetiger 7 . Chaetiger 9 with low pre- and postchaetal ridges, and distinct digitiform lateral lobes. Thoracic chaetigers with simple capillary chaetae. Abdominal lateral lamellae triangular, with pointed tips. Hooded hooks tridentate, vis-á-vis in two facing groups. Pouches (C configuration) present posterior to chaetiger 18 or 19 (see Remarks, below).

Description. A stout species; thoracic width of holotype (anterior fragment only) similar to abdomen, with marked constriction between thorax and abdomen at chaetiger 9 (Fig. 5A). Thoracic width $0.8 \mathrm{~mm}$, increasing to $1.15 \mathrm{~mm}$ around chaetigers 5-7, approximately $6 \mathrm{~mm}$ long (including prostomium). Chaetigers characteristically bulbous, rounded, total length approximately $11 \mathrm{~mm}$ (shorter than $16 \mathrm{~mm}$, as described by both Ehlers and Wilson) for 19 chaetigers. Specimen in fairly poor condition, appearing poorly preserved and thus very fragile, as previously reported by Wilson (1958) who stated that "the specimen shows every indication of having been partially flattened." Chaetiger 16 previously dissected.

Prostomium roughly as wide as long ( $0.91 \mathrm{~L}: \mathrm{W}$ ratio), subtriangular, anterior margin straight and smooth, with rudimentary horns (Figs. 5A-B). Two inner dorsal muscular ridges, diverging anteriorly, two outer ridges abutting inner ridges. Exact nature of muscular ridges difficult to discern due to condition of specimen. Proboscis everted, heart-shaped, ridged inferiorly and superiorly. Only left-hand palp retained, arising ventrolaterally from base of prostomium, short but incomplete, reaching to around chaetigers 5-6. Palp papillated for the most part, nonpapillated region reaching to chaetiger 3, papillae initially short, becoming longer distally, 2-3 rows of papillae either side of inconspicuous ventral groove. Peristomium of roughly equal size to chaetiger 1.

Notopodia of chaetigers 1-8 similar (Figs. 5C, E, G, I-J); low triangular notopodial prechaetal ridges confluent with triangular postchaetal lamellae that terminate in pointed tips, decreasing in size along thorax. Notopodial lamellae shorter than neuropodial. Anterior neuropodial postchaetal lamellae scoop-shaped, initially ventral in position, distally expanded, especially on chaetiger 1 (Figs. 5C-D), but decreasing in size to chaetiger 4 (Figs. 5C-H). Small pointed tips present on chaetigers 2-4. Neuropodial lamellae further reduce on chaetigers 5-6, becoming shorter and narrower (Fig. 5I). Neuropodial lamellae on chaetigers 7-8 larger, triangular, and entirely postchaetal (Fig. 5J). No superior processes (DML) on thoracic chaetigers.

Chaetiger 9 (Fig. 5K) lamellae similar in both rami, with low pre- and postchaetal ridges and distinct digitiform lateral lobes. All thoracic chaetae simple capillaries, those of chaetiger 9 about $50-60 \%$ the length of those on preceding chaetigers.

Abdominal chaetigers with sharply-pointed triangular shaped lateral lamellae, of about equal size in both rami (Figs. 5L-M). No dorsal (DML) or ventral (VML) processes on abdominal chaetigers observed. Abdominal chaetae tridentate hooded hooks, of similar size. Hooks in two groups, main fangs vis-á-vis. About 12-14 hooks per ramus in first four abdominal chaetigers, with approximately five hooks next to lamella and nine in lateral group, decreasing in number to approximately 10 (4 and 6) in the fifth abdominal chaetiger. Hooks not illustrated in order to prevent further damage to the specimen. No lateral pouches observed on holotype. Posterior unknown.

Color. Preserved holotype cream-white in alcohol. Thoracic pigment band no longer visible, however chaetigers 5-9 appear more opaque than preceding chaetigers. Methyl green staining thorax lightly all over, with light transverse bands both dorsally and ventrally between chaetigers 3 and 4 and dorsally between 6-9. Sparse globular white speckles dorsally between chaetigers 3 and 4 (Fig. 
5A). Scattered pigment interparapodially in abdomen, and two longitudinal lines either side of the mid-ventral line, starting from chaetiger 9.

Remarks. When commenting on Ehlers' figures, Wilson (1958) stated "his drawings and descriptions are not entirely satisfactory (Wesenberg-Lund 1949, p. 330, is in agreement) and lack proper figures of parapodia." Wilson's reexamination was intended to clarify the difference between M. cincta and M. alleni Wilson, 1958. However, although it added information to the original description, it did not adequately describe or illustrate the type, particularly with reference to the thoracic lamellae. The redescription provided herein rectifies this situation. In addition all known records are herein discussed.

A stout anterior fragment collected by the University of Cape Town (BMNH.1955.4.1.96) was examined. The specimen was $12 \mathrm{~mm}$ long for 30 chaetigers and constricted at the ninth chaetiger. A curved fragment of 11 chaetigers and $5 \mathrm{~mm}$ in length became detached during examination. Another $4 \mathrm{~mm}, 10$ chaetiger, fragment and a palp were already present in the specimen tube. The thorax (including prostomium) measured $3.2 \mathrm{~mm}$ long and $0.9 \mathrm{~mm}$ wide at chaetiger 1 , and $1.2 \mathrm{~mm}$ wide at chaetiger 7. The specimen was in reasonable condition, with lamellae generally well preserved. The prostomium was slightly indented medially, with the two outer ridges less distinct than the inner ridges and no distinct muscular pattern laterally. The palps had short nonpapillated regions, were robust and possessed short papillae, more akin to the Hong Kong Magelona cf. cincta specimens (see below), than to the holotype. Neuropodial lamellae of chaetiger 1 were slightly more rounded distally, with a less pointed tip than on the holotype; however, the anterior chaetigers otherwise agree well with the type specimen. Abdominal hooded hooks tridentate, secondary teeth small as figured by Ehlers (1908: fig. 12); however, the main fang is more robust with a generally more rounded tip, similar to those figured for the Hong Kong material (Fig. 6M-O). The specimen possesses posteriorly open C-configuration pouches on the following chaetigers: 19L, 21R, 24L, 30L and $30 \mathrm{R}$. The separate fragment has pouches on the $3^{\text {rd }}$ chaetiger left and right, $5^{\text {th }}$ chaetiger left, $6^{\text {th }}$ chaetiger right, $8^{\text {th }}$ left and $10^{\text {th }}$ right, but as the exact position of this fragment cannot be determined, chaetiger numbers cannot be assigned. A sheath-like, multilayered tube covered in sand grains is present (now partially removed to facilitate observation of the abdominal chaetigers), this tube is similar to that seen on some specimens of $M$. alleni.

The coloration of the specimen is brown, darker in the abdomen, with brown interparapodial pigmentation. It also has a distinct red pigment band between chaetigers 5-8, as originally described for the type and carries the same white speckled pigmentation as seen in the holotype. This specimen was previously examined by Wilson (1958), and we agree with him in regarding it as M. cincta. Day (1967: 495) was the first to record the presence of pouches in this species.

Kirkegaard (1959) reported $M$. cincta from off French Guinea (Sta. 45, $9^{\circ} 23^{\prime} \mathrm{N}, 15^{\circ} 07^{\prime} \mathrm{W}$ ), collected during the Atlantide Expedition in 1945. One of the three specimens was deposited in the collections of the Natural History Museum, London (BMNH 1961.15.21), and has been examined. The prostomium and parapodial lamellae in the thorax and abdomen are markedly different to those of $M$. cincta, and it is likely to represent an undescribed species. Wilson (1958) states that specimens previously attributed to $M$. cincta from the Firth of Clyde, and those housed within the British Museum collections from Dogger Bank (North Sea) and Plymouth are in fact Magelona alleni.

Magelona cincta has additionally been reported off the Northwest Indian Shelf (Jayaraj et al. 2007), the Natuna Islands, South China Sea (Al-Hakin \& Glasby 2004) and inner Argolikos Bay, Southeastern Peloponnese, Greece (Makra \& Nicolaidou 2000). 


\section{Magelona cf. cincta}

Figure 6

Material examined. Hong Kong (NE), Chek Mun Hoi Hap (Tolo Channel): east headland of Fung Wong Wat on north side of outer Channel opposite Heung Lo Kok (Gruff Head), Sta. 86 of Erséus (1990), silt-clay-shell, 9 m (NMW.Z.1986.079.0177; af), diver coll. A.S.Y. Mackie \& P. G. Oliver 13 Apr 1986; Hoi Ha Wan (Jones Cove), southwest off Flynn Point (Sample 1 = Sta. 15 of Erséus 1992), silty sand with many burrows (crustacean?), 9 m (NMW.Z.1989.117.0046; c), diver coll. A.S.Y. Mackie \& P. Sundberg 12 Apr 1989.- - Tai Pang Wan (Mirs Bay), east side of Wang Mun Hoi (Deep Pass) between Kat O Chau (Crooked Island) and Ngo Mei Chau (Crescent Island), Sta. 59 of Erséus (1990), shell gravel with silt clay, 13 m (NMW.Z.1986.079.0178; af), diver coll. for Prof. Erséus 7 Apr 1986; east of Tan Ka Wan, Sta. 2, fine silt/clay, 24 m (NMW.Z.1986.079.0179; af), coll. A. Hirayama 5 Apr 1986; north of Shek Ngau Chau (Breaker Reef: $22^{\circ} 28^{\prime} \mathrm{N}, 114^{\circ} 25^{\prime} \mathrm{E}$ ), Sta. 55 of Erséus (1990), shell debris, silt-clay, 21 m (NMW.Z.1986.079.0176; af), diver coll. for Prof. Erséus 6 Apr 1986.

Diagnosis. A stout species, prostomium wider than long, sub-trapezoidal, with rudimentary horns. Palps robust, with long papillae. Chaetigers 1-8 with slender, smooth-edged, triangular notopodial postchaetal lamellae with pointed tips, no superior processes present. Ventral neuropodial lamellae below chaetae anteriorly, becoming postchaetal from chaetigers 5-6. Neuropodial lamellae of first two chaetigers flat, scoop-shaped, distally expanded. Chaetiger 9 similar in both rami, low ridges with short triangular lobes. Thoracic chaetigers with simple capillary chaetae. Abdominal lateral lamellae sinuous leaf-shaped with pointed tips. Hooded hooks tridentate, in two facing groups (vis-á-vis). C-configuration pouches on abdominal chaetigers.

Description. A large, stout species; thoracic width similar to abdomen, no clear constriction between thorax and abdomen. Complete specimen (NMW.Z.1989.117.0046; in two fragments; anterior fragment 36 chaetigers, posterior fragment 16 chaetigers); thorax (including prostomium) $1.8 \mathrm{~mm}$ long, $0.45 \mathrm{~mm}$ at maximum width (thorax widest between chaetigers 5-8), total length $7 \mathrm{~mm}$ for 52 chaetigers. Median thoracic chaetigers characteristically rounded and bulbous laterally.

Prostomium wider than long (0.6-0.75 L:W ratio), subtrapezoidal, anterior margin smooth, rudimentary horns (Figs. 6A-B); two prominent inner longitudinal dorsal muscular ridges, proximal but separated for entirety, anteriorly divergent and extending into the prostomial corners. Two outer muscular ridges abutting inner ridges, no other obvious prostomial ornamentation. Prostomium of complete specimen damaged laterally giving the appearance of a much wider prostomium (Fig. 6A); prostomial shape better represented in other material (Fig. 6B). Proboscis partially everted, oval, weakly ridged. Only left-hand palp retained (now detached), arising ventrolaterally from base of prostomium, short, reaching approximately chaetiger 12. Palps papillated almost to base (nonpapillated region reaching to chaetiger 1-2), with 6-8 rows of papillae proximally, decreasing to two distally (papillae very long, length decreasing only at proximal end). Peristomium 1.5 times longer than chaetiger 1.

Notopodia of chaetigers 1-8 similar (Figs. 6C-H); low triangular notopodial prechaetal ridges confluent with slender triangular postchaetal lamellae that terminate in pointed tips, decreasing in size from chaetigers 1-6, larger on 7-8, no superior processes (DML) present. Neuropodial lamellae (VNL) ventral in position, initially scoop-shaped, decreasing in size from chaetigers 1-5, but larger on chaetigers $6-8$, becoming slender cirriform, and postchaetal by chaetigers $5-6$. Neuropodial lamellae of chaetigers 1 and 2 slightly distally expanded, with small tips. 

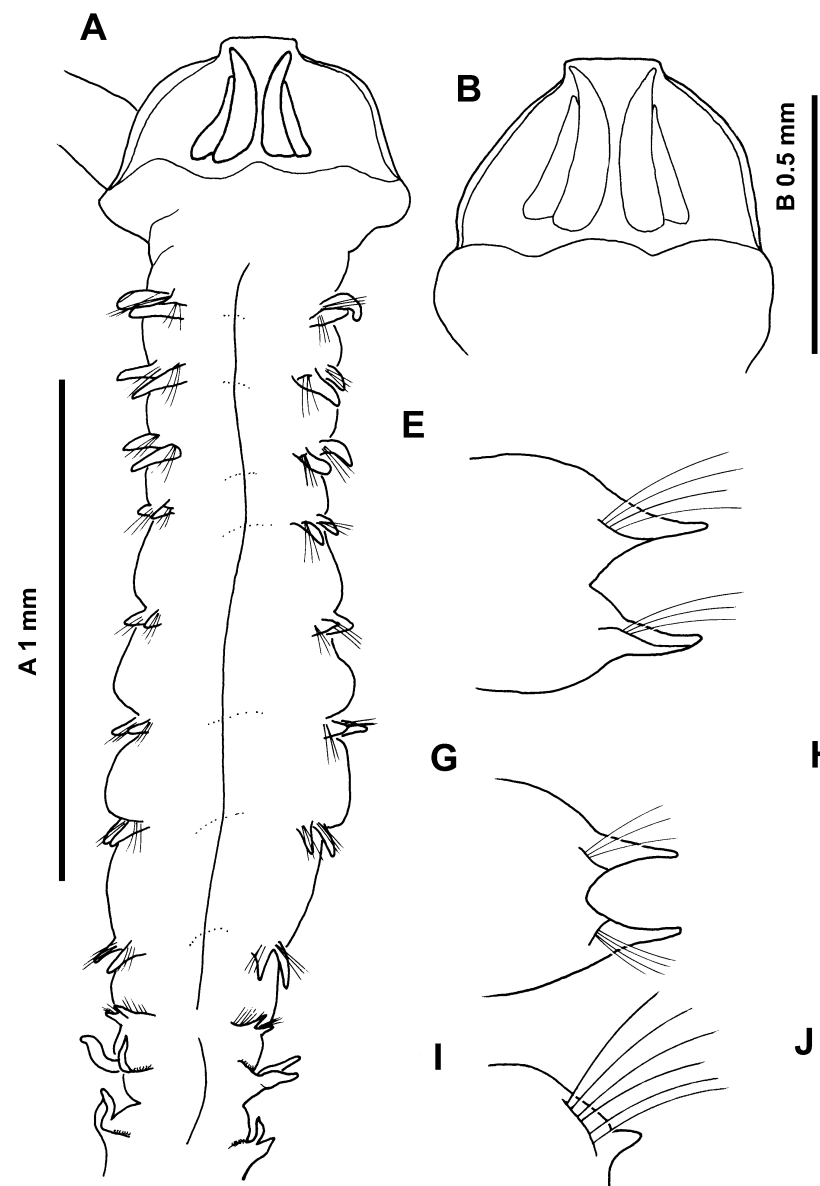

C

E

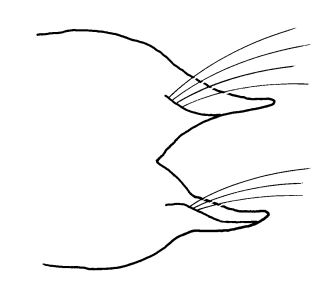

G
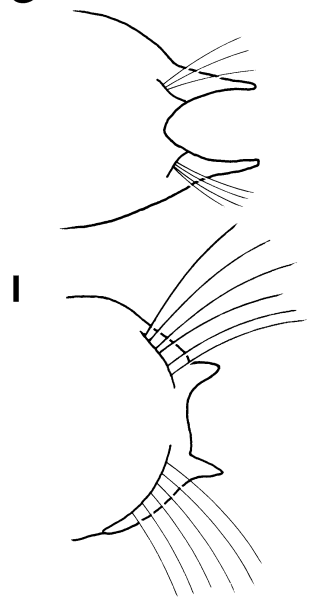

K

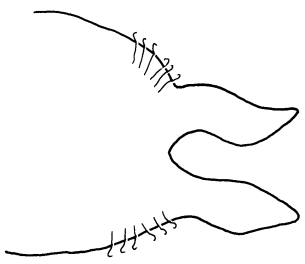

M

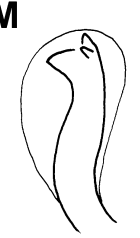

$\mathbf{F}$
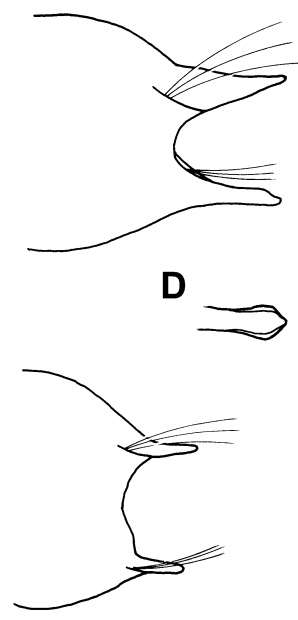

H
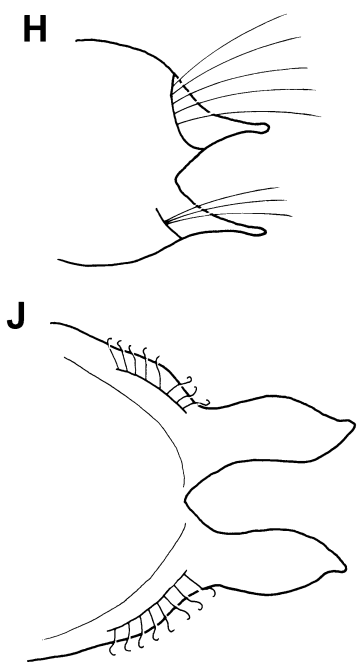

$\mathbf{N}$
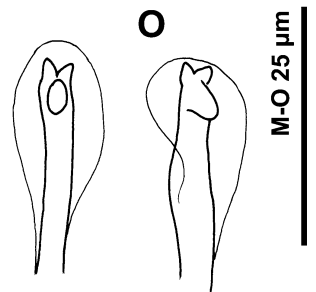

Chaetiger 9 (Fig. 6I) lamellae similar in both rami, postchaetal ridges, lateral sub-triangular lobes. All thoracic chaetae simple capillaries.

FIGURE 6. Hong Kong Magelona cf. cincta (Anterior, posterior, and parapodia, NMW.Z.1989.117.00046, Prostomium, NMW.Z.1986.079.0177). A, anterior region, dorsal view; B, prostomium, dorsal view; C, chaetiger 1, 
anterior view; D, neuropodial lamellae chaetiger 1, dorsal view; E-K, chaetigers 3, 5, 7, 8, 9, 12, and 25 respectively, anterior views; L, posterior, dorsal view; $\mathrm{M}-\mathrm{O}$, tridentate hooded hooks, lateral, frontal and oblique frontal views respectively.

Abdominal chaetigers with sharply pointed, rather sinuous leaf-shaped lateral lamellae, of about equal size in both rami (Figs. 6J-K). No dorsal (DML) or ventral (VML) processes observed on abdominal chaetigers. Abdominal chaetae tridentate hooded hooks, of similar size (Figs. 6M-O). Hooks in two groups, main fangs vis-á-vis. Initially approximately eight hooks per ramus, decreasing to six on median and postchaetal chaetigers. Unpaired lateral pouches ( $\mathrm{C}$ configuration) present on abdominal segments. Complete specimen with pouches on 22L, 23R, 26L, 30R, 31L, 41R, 43L, 44R, 46L, and 47R. Pygidial cirri absent, presumed missing, although with no obvious signs of damage. Anus terminal, appearing slightly ventral, surrounded dorsally and laterally by small ridge.

Color. Preserved specimens cream-white in alcohol, weak pigment band present between chaetigers 5-8, difficult to discern in some specimens. Methyl green staining showed no obvious pattern dorsally, weakly stained all over and a few speckles interparapodially, with darker pigmentation between chaetigers 5-8. Ventrally, light pigmentation as a transverse band between chaetigers 2-3 and two longitudinal bands either side of the mid-ventral line from chaetiger 9 to the pygidium. Light interparapodial patches in abdomen.

Patches of white speckles seen dorsally proximal to parapodia, between chaetigers $1-5$, and ventrally as transverse bands between chaetigers 3-4. White speckles either side of the mid-ventral line on abdomen.

Habitat. Found at five stations in sandy mud and muddy shelly sediments, 9-21 m.

Remarks. Magelona cf. cincta shares several similarities with the holotype of M. cincta (see above). Both possess scoop-shaped, distally expanded neuropodial lamellae on chaetigers 1-2, characteristic rounded and laterally bulbous median thoracic chaetigers, and the presence of small white bulbous "papillae" dorsally on thoracic chaetigers (seen especially with methyl green staining). The Hong Kong material differs in: (1) the nature of the neuropodial lamellae of chaetiger 1, being less expanded distally, (2) the palps, which are more robust, have long papillae and a much shorter non-papillated region, and (3) the abdominal lamellae, which are less triangular and narrower basally.

Nateewathana \& Hylleberg (1991) recorded Magelona cincta from Thailand and their drawings appear to correspond to the Hong Kong material collected in our survey. However, they stated that their specimens differed from $M$. cincta in not possessing lateral pouches, first described as present in $M$. cincta by Day (1967). The holotype of $M$. cincta is much larger than the Hong Kong material. It may be possible in the future to separate these two species with more material from Hong Kong and South Africa. Nevertheless, the perceived differences between the Hong Kong material and the holotype could be caused by both size and preservation technique, and therefore it is not considered a separate species at the present time.

Magelona cf. cincta also approaches a suite of five species including M. japonica Okuda, 1937, M. koreana Okuda, 1937 (originally M. japonica var. koreana; see Jones 1971), M. alleni, M. equilamellae Harmelin, 1964, and M. symmetrica Mortimer \& Mackie, 2006, based on the shape of both thoracic and abdominal lamellae. However, all lack scoop-like distally expanded anterior neuropodial lamellae.

\section{Discussion}

The present study has highlighted the need for close examination of all aspects of magelonid morphology. The newly discovered morphological similarities between $M$. cornuta and $M$. 
crenulifrons, species that can co-occur within a location, have extended our understanding of both species and raised the prospect that other "widely" distributed species may also represent species complexes. This could well be the case for M. cincta. A large specimen from Mozambique was found to agree well with the similarly sized holotype. However, smaller specimens from Hong Kong exhibited some differences in the shapes of the parapodial lamellae. The significance of these observations can only be properly resolved through the study of additional material (preferably population samples involving different sized individuals) from southern Africa and elsewhere.

\section{Acknowledgements}

We would like to acknowledge Danny Eibye-Jacobsen (Zoological Museum, University of Copenhagen), Leslie Harris (Natural History Museum of Los Angeles County), and Birger Neuhaus (Museum für Naturkunde der Humboldt-Universität zu Berlin) for their help and loan of type material. Roger Bamber and Emma Sherlock at the Natural History Museum, London, and Paul Shin and his student Nelson Lam at the City University of Hong Kong who kindly made available additional material. The second author thanks Professor Brian Morton for inviting him to the Second and Fourth International Marine Biological Workshops, during which much of the Hong Kong material was collected. Christer Erséus, Akira Hirayama, and Paul Shin shared their workshop samples, and Graham Oliver and Per Sundberg helped with the collecting. We would like also to thank the National Museum Wales for funding to carry out collection of specimens.

\section{References}

Aguirrezabalaga, F., Ceberio, A. \& Fiege, D. (2001) Octomagelona bizkaiensis (Polychaete: Magelonidae) a new genus and species from the Capbreton Canyon (Bay of Biscay, north-east Atlantic). Journal of the Marine Biological Association of the United Kingdom, 81, 221-224.

Al-Hakin, I. \& Glasby, C.J. (2004) Polychaeta (Annelida) of the Natuna Islands, South China Sea. The Raffles Bulletin of Zoology, Supplement 11, 25-45.

Amoureux, L. (1983) Annélides polychètes du golfe d'Aqaba (mer Rouge). Description d'un genre nouveau et de deux espèces nouvelles. Bulletin du Muséum National d'Histoire Naturelle, Paris, Série 4, 5, 723-742.

Cunningham, J.T. \& Ramage, G.A. (1888) The Polychaeta Sedentaria of the Firth of Forth. Transactions of the Royal Society of Edinburgh, 33, 635-684.

Ehlers, E. (1908) Die bodensässigen Anneliden aus dem Sammlungen der deutschen Tiefsee-Expedition. Wissenschaftliche Ergebnisse der Deutschen Tiefsee-Expedition auf dem Dampfer "Valdivia" 1898-1899, 16, $1-167$.

Erséus, C. (1990) Marine Oligochaeta of Hong Kong. In: Morton, B. (Ed.) Proceedings of the Second International Marine Biological Workshop: The Marine Flora and Fauna of Hong Kong and Southern China, Hong Kong, 2-24 April 1986. Hong Kong University Press, Hong Kong, pp. 259-335.

Erséus, C. (1992) Oligochaeta from Hoi Ha Wan. In: Morton, B. (Ed.) Proceedings of the Fourth International Marine Biological Workshop: The Marine Flora and Fauna of Hong Kong and Southern China, Hong Kong, 11-29 April 1989. Hong Kong University Press, Hong Kong, pp. 909-917.

Fiege, D., Licher, F. \& Mackie, A.S.Y. (2000) A partial review of the European Magelonidae (Annelida: Polychaeta): Magelona mirabilis redefined and M. johnstoni sp. nov. distinguished. Journal of the Marine Biological Association of the United Kingdom, 80, 215-234.

Gallardo, V.A. (1968) Polychaeta from the Bay of Nha Trang, South Viet Nam. Naga Report, 4(3), 35-279.

Gibson, R. (1990) The macrobenthic nemertean fauna of Hong Kong. In: Morton, B. (Ed.) Proceedings of the 
Second International Marine Biological Workshop: The Marine Flora and Fauna of Hong Kong and Southern China, Hong Kong, 2-24 April 1986. Hong Kong University Press, Hong Kong, pp. 33-212.

Harmelin, J.G. (1964) Étude de l'endofaune des "mattes" d'herbiers de Posidonia oceanica Delile. Recueil des Travaux de la Station Marine d'Endoume, 35(51), 43-105.

Hartman, O. (1974) Polychaetous annelids of the Indian Ocean including an account of species collected by members of the International Indian Ocean Expeditions, 1963-1964 and a catalogue and bibliography of the species from India. Journal of the Marine Biological Association of India, 16, 191-252.

Hartman, O. (1976) Polychaetous annelids of the Indian Ocean including an account of species collected by members of the International Indian Ocean Expeditions, 1963-1964, and a catalogue and bibliography of the species from India. Part II. Journal of the Marine Biological Association of India, 16, 609-644.

Hernández-Alcántara, P. \& Solís-Weiss, V. (2000) Magelonidae from the Mexican Pacific and northern Gulf of Mexico, with the description of a new genus (Meridithia) and four new species. In: Reish, D.J. \& Lana, P. (Eds.), Proceedings of the 6th International Polychaete Conference, Curitiba, Brazil, 1998. Bulletin of Marine Science, 67, 625-644.

Hirayama, A. (1990) Marine gammaridean Amphipoda (Crustacea) from Hong Kong I. The Family Corophiidae, Genus Corophium. In: Morton, B. (Ed.) Proceedings of the Second International Marine Biological Workshop: The Marine Flora and Fauna of Hong Kong and Southern China, Hong Kong, 2-24 April 1986. Hong Kong University Press, Hong Kong, pp. 449-485.

Hylleberg, J. \& Nateewathana, A. (1991) Temporal and spatial distribution of subtidal magelonid polychaetes at Phuket Island, Thailand, Andaman Sea. In: Petersen, M.E. \& Kirkegaard, J.B. (Eds.) Systematics, biology and morphology of world Polychaeta. Proceedings of the 2nd International Polychaete Conference, Copenhagen, 1986. Ophelia, Suppl. 5, 573-578.

Intes, A. \& Le Loeuff, P. (1975) Les annélides polychètes de côte d'Ivoire. I. - Polychètes errantes- compte rendu systématique. Cahiers de l'Office de la Recherche Scientifique et Technique Outre-Mer, Série Océanographie, 13(4), 267-321.

Jayaraj, K., Jayalakshmi, K. \& Saraladevi, K. (2007) Influence of Environmental Properties on Macrobenthos in the northwest Indian shelf. Environmental Monitoring and Assessment, 127(1-3), 459-475.

Johnson, H. (1901) The Polychaeta of the Puget Sound region. Proceedings of the Boston Society of Natural History, 29, 381-437.

Jones, M.L. (1963) Four new species of Magelona (Annelida, Polychaeta) and a redescription of Magelona longicornis Johnson. American Museum Novitates, 2164, 1-31.

Jones, M.L. (1971) Magelona berkeleyi n.sp. from Puget Sound (Annelida: Polychaeta) with a further redescription of Magelona longicornis Johnson and a consideration of recently described species of Magelona. Journal of the Fisheries Research Board of Canada, 28, 1445-1454.

Jones, M.L. (1978) Three new species of Magelona (Annelida, Polychaeta) and a redescription of Magelona pitelkai Hartman. Proceedings of the Biological Society of Washington, 91, 336-363.

Kirkegaard, J.B. (1959) The Polychaeta of West Africa Part I. Sedentary species. Atlantide Report 5, 7-117.

Mackie, A.S.Y. (1990) The Poecilochaetidae and Trochochaetidae (Annelida: Polychaeta) of Hong Kong. In: Morton, B. (Ed.) Proceedings of the Second International Marine Biological Workshop: The Marine Flora and Fauna of Hong Kong and Southern China, Hong Kong, 2-24 April 1986. Hong Kong University Press, Hong Kong, pp. 337-362.

Mackie, A.S.Y., Oliver, P.G. \& Kingston, P.F. (1993) The macrobenthic infauna of Hoi Ha Wan and Tolo Channel, Hong Kong. In: Morton, B. (Ed) The Marine Biology of the South China Sea. Proceedings of the First International Conference on the Marine Biology of Hong Kong and the South China Sea, Hong Kong 28 October-3 November 1990. Hong Kong University Press, Hong Kong, pp. 657-674.

Makra A. \& Nicolaidou, A. (2000) Benthic communities of the inner Argolikos Bay. Belgian Journal of Zoology, 130 Supplement 1, 61-67.

Mortimer, K. \& Mackie, A.S.Y. (2003) The Magelonidae (Annelida: Polychaeta) from the Seychelles, with the description of three new species. In: Sigvaldadóttir, E., Mackie, A.S.Y., Helgason, G.V., Reish, D.J., 
Svavarsson, J., Steingrímsson, S.A. \& Gudmundsson, G. (Eds) Advances in Polychaete Research. Hydrobiologia, 496 (1-3), 163-173.

Mortimer, K. \& Mackie, A.S.Y. (2006) The Magelonidae (Annelida: Polychaeta) from the Seychelles. 2. Description of four additional species, three new to science. In: Sardá, R., San Martín, G., López, E., Martin, D. \& George, D. (Eds) Scientific Advances in Polychaete Research. Scientia Marina. 70(S3), 125-137.

Müller, F. (1858) Einiges über die Annelidenfauna der Insel Santa Catharina au der brasilianischen Küste. Archiv Für Naturgeschicthe, 24, 211-220.

Nateewathana, A. \& Hylleberg, J. (1991) Magelonid polychaetes from Thailand, the Andaman Sea, with descriptions of eight new species. In: Petersen, M.E. \&. Kirkegaard, J.B. (Eds) Systematics, biology and morphology of world Polychaeta. Proceedings of the 2nd International Polychaete Conference, Copenhagen, 1986. Ophelia, Suppl. 5, 169-184.

Okuda, S. (1937) Spioniform polychaetes from Japan. Journal of the Faculty of Science, Hokkaido University, Series 6 (Zoology), 5, 217-254.

Shin, P.K.S. (1982a) The macrobenthic infauna of Tolo Harbour and Tolo Channel, Hong Kong. In: Morton, B. \& Tseng, C.K. (Eds) Proceedings of the First International Marine Biological Workshop: The Marine Flora and Fauna of Hong Kong and Southern China, Hong Kong 1980. Hong Kong University Press, Hong Kong, pp. 721-731.

Shin, P.K.S. (1982b) Some polychaetous annelids from Hong Kong waters. In: Morton, B. \& Tseng, C.K. (Eds) Proceedings of the First International Marine Biological Workshop: The Marine Flora and Fauna of Hong Kong and Southern China, Hong Kong 1980. Hong Kong University Press, Hong Kong, pp. 161-172.

Shin, P.K.S. (1990) Benthic invertebrate communities in Tolo Harbour and Mirs Bay: a review. In: Morton, B. (Ed.) Proceedings of the Second International Marine Biological Workshop: The Marine Flora and Fauna of Hong Kong and Southern China, Hong Kong, 2-24 April 1986. Hong Kong University Press, Hong Kong, pp. 883-898.

Shin, P.K.S. (1998) Biodiversity of subtidal benthic polychaetes in Hong Kong coastal waters. In: Morton, B. (Ed) The Marine Biology of the South China Sea. Proceedings of the Third International Conference on the Marine Biology of Hong Kong and the South China Sea, Hong Kong, 28 October-1 November 1996. Hong Kong University Press, Hong Kong, pp. 57-74.

Shin, P.K.S. (2003) Changes in benthic infaunal communities in Tolo Harbour: will the trend continue? In: Morton, B. (Ed.) Perspectives on Marine Environment Change in Hong Kong and Southern China, 1977-2001. Proceedings of an International Workshop Reunion Conference, Hong Kong 21-26 October 2001. Hong Kong University Press, Hong Kong, pp. 579-592.

Shin, P.K.S. \& Thompson, G. B. (1982) Spatial distribution of the infaunal benthos of Hong Kong. Marine Ecology Progress Series, 10, 37-47.

Uebelacker, J.M. \& Jones, M.L. (1984) Family Magelonidae. In: Uebelacker, J.M. \& Johnson, P.G. (Eds), Taxonomic Guide to the Polychaetes of the Northern Gulf of Mexico. Final Report to the Minerals Management Service, contract 14-12-001-29091. Barry A. Vittor \& Associates, Mobile, Alabama, pp. 7.1-7.29.

Wesenberg-Lund, E. (1949) Polychaetes of the Iranian Gulf. Danish Scientific Investigations in Iran, 4, 247-400.

Wilson, D.P. (1958) The polychaete Magelona alleni n. sp. and a re-assessment of Magelona cincta Ehlers. Journal of Marine Biological Association of the United Kingdom, 37, 617-626.

Wilson, D.P. (1959) The polychaete Magelona filiformis sp. nov. and notes on other species of Magelona. Journal of the Marine Biological Association of the United Kingdom, 38, 547-556. 
200 - Zoosymposia 2 @ 2009 Magnolia Press 